Meta

Journal des tradlucteurs

Translators' Journal

\title{
Lexique ferroviaire / Railway Lexicon
}

\section{Paul A. Horguelin}

Volume 13, numéro 2, juin 1968

URI : https://id.erudit.org/iderudit/017812ar

DOI : https://doi.org/10.7202/017812ar

Aller au sommaire du numéro

Éditeur(s)

Les Presses de l'Université de Montréal

ISSN

0026-0452 (imprimé)

1492-1421 (numérique)

Découvrir la revue

Citer cet article

Horguelin, P. A. (1968). Lexique ferroviaire / Railway Lexicon. Meta, 13(2),

67-67. https://doi.org/10.7202/017812ar d'utilisation que vous pouvez consulter en ligne.

https://apropos.erudit.org/fr/usagers/politique-dutilisation/ 


\section{PROBLÈMES ET SOLUTIONS}

\section{LEXIQUE FERROVIAIRE / RAILWAY LEXICON}

Le Service des relations extérieures et de la publicité du Canadien Pacifique a publié une deuxième édition, revue et corrigée, de son Lexique ferroviaire. On y trouve un bon vocabulaire de base qui englobe la terminologie technique et administrative propre aux chemins de fer. Les termes proposés dénotent une recherche constante de l'équivalent exact, écartant le calque ou la création hybride - ce dont on ne peut que féliciter l'auteur, M. Lorenzo Côté.

Le vocabulaire des chemins de fer est à l'occasion très imagé, comme le prouvent les termes suivants: crocodile (automatic stop), reniflard (blow-valve), crapaud (clip), rail à double champignon (bullhead rail), locomotive haut-le-pied (light engine); on se demande toutefois, avec une certaine inquiétude, ce que peut bien être un dispositif d'homme mort (deadman's handle) ... Certaines équivalences nous ont paru particulièrement intéressantes: siffler au disque (to wait for an opening), bagages accompagnés (luggage travelling with the passenger), tarif aiguillon (incentive rates), et on pourrait philosopher sur les raisons qui poussent un francophone à nommer voie descendante ce que l'anglophone appelle une up line.

On peut regretter que dans certains cas le terme anglais soit suivi de plusieurs équivalents français qui ne sont pas synonymes (ex., branch = branche, succursale, filiale), le choix du terme exact - en fonction du contexte - étant laissé au jugement du lecteur. Il faut dire cependant que c'est l'exception, et le sens des mots ayant une large aire sémantique est généralement précisé; par exemple, adjust : régler, ajuster (une balance, des freins, une montre, un compas); caler (les balais d'une dynamo); monter (un appareil); agencer (les parties d'une machine); étalonner (un instrument); rectifier, centrer (un outil); tarer (une soupape); mettre au point (un microscope, un moteur); égaliser (la pression). Notons aussi Porthographe «insulaire» de certains mots (tyre) et, plus insolite, le choix du terme britannique petrol meter.

Les réserves que l'on pourrait apporter au Lexique ferroviaire, par ailleurs 
irréprochable, ne touchent que des questions de détail. Nous avons relevé quelques fautes de typographie: clef à malette (molette), détenteur de pression (détendeur), train no. $22\left(\mathrm{n}^{\circ}\right)$, et quelques traits d'union superflus (papier-journal; non-disponible, non-réglable). On pourrait également mettre en doute la légitimité de certains termes donnés comme seuls équivalents (ex., superintendent: surintendant) ou placés en concurrence avec d'autres (ex., snow-plow : chasse-neige, charrue à neige; traffic manager : directeur, gérant du trafic; local : local, régional, de la place; heavy duty : dur service, grand rendement, haute capacité, grande puissance, fort débit).

À part ces quelques réserves sans grande importance, le Lexique ferroviaire présente un bon vocabulaire de vulgarisation avec lequel cheminots et employés des chemins de fer auraient avantage à se familiariser. Sachant que crate est une caisse à claire-voie, le préposé aux bagages de la gare maritime de Québec ne demandera peut-être plus à l'immigrant qui désire expédier une motocyclette à Montréal «Faut-y vous la crêter?»

Paul A. Horguelin

\section{RÉFLEXIONS SUR QUELQUES TERMES DE CINÉMA E'T DE TÉLÉVISION}

Parmi les sources où s'alimente le vocabulaire technique, l'usage n'est pas la moindre. Encore faut-il ne pas renoncer à utiliser son jugement devant les diktats de ce maître, faillible comme nous tous. L'usage souffre d'être dirigé. Dans le domaine technique, peut-être plus qu'ailleurs. Car le vocabulaire technique doit se développer rapidement au gré des circonstances, selon la fantaisie des usagers, en subissant l'influence d'autres langues et d'autres techniques.

Les droits de l'usage sont fonction de son ancienneté et de l'autorité de ceux qui le propagent. Quand un usage compte quelques lustres d'existence, c'est peine perdue que de vouloir le déraciner. De même quand les théoriciens, les critiques et les techniciens sont unanimes à employer une expression qu'on pourrait juger répréhensible, il y a fort à parier que cette "faute » deviendra "règle ".

Il est très difficile, par exemple, de déloger de la langue du cinéma et de la télévision le terme travelling ${ }^{1}$ qui a cours dans la technique cinématographique depuis au moins quarante ans ${ }^{2}$. Le mot a d'ailleurs provigné par composition: on trouve aujourd'hui travelling-avant (dolly-in), travelling-arrière (dolly-out), travelling latéral (lateral follow shot), travelling vertical (vertical follow shot). C'est un mot qu'il faut ranger au nombre des emprunts regrettables mais inévitables.

Il ne faudrait pas cependant céder ainsi devant tous les anglicismes qui contaminent la langue des studios de radiotélévision.

Pourquoi préférer back screen projection à projection par transparence qu'on peut facilement abréger en transparence? Pourquoi la désignation des plans de prise de vues se fait-elle le plus souvent en anglais en dépit d'un usage reçu en français universel depuis assez longtemps? Pourquoi les procédés de transition et

1. Prise de vue au moyen d'une caméra montée sur un chariot.

2. Cf. Jean Giraud, le Lexique trançais du cinéma des origines à 1930, Paris, Centre national de la recherche scientifique, 1958, p. 201. 